\title{
2
}

\section{Caracterización de los sistemas de producción caprinos, en la provincia de Santa Elena.}

Julio Villacrés Matías, Lourdes Ortega Maldonado, Debbie Chávez García

Recibido: marzo 2017 Aprobado: mayo 2017 


\title{
Caracterización de los sistemas de producción caprinos, en la provincia de Santa Elena
}

\section{Characterization of caprine production systems, in the province of Santa Elena}

\author{
Julio Villacrés Matías ${ }^{(1)}$, Lourdes Ortega Maldonado ${ }^{(2)}$, Debbie Chávez García ${ }^{(3)}$ \\ Facultad de Ciencias Agrarias \\ Universidad Estatal Península de Santa Elena (UPSE) ${ }^{(1)(2)(3)}$ \\ jvillacresk8@ hotmail.es
}

\begin{abstract}
Resumen
La provincia de Santa Elena es reconocida como productora de ganado caprino, ya sea por los sistemas de producción existentes, así como también por el plato típico que de ésta se oferta; la inexistencia de estudios que describan los sistemas de producción caprinos existentes en Santa Elena, ha sido el principal motivo de este estudio; que consistió en el levantamiento de información primaria aplicando encuestas a ganaderos de todas las comunas de la provincia; que fueron análizada mediante estadística descriptiva, donde se encuesto un total de 497 ganaderos, los cuales contaban con un total de 7293 animales, siendo el $75 \%$ animales hembras, lo que demuestra una buena relación de la composición del hato, que además, está conformado con un 92 \% de cabras criollas. Las variables analizadas estan relacionadas con la producción actual de caprinos y fueron agrupadas en varios componentes que son, componente social, composición del hato, tecnología existente, características de la alimentación, sanidad animal y comercialización.
\end{abstract}

Palabras Claves: Ganado, Hato, Producción, Cabras.

\begin{abstract}
The province of Santa Elena is recognized as a producer of goats, either by the existing production systems, as well as by the typical dish that is offered; the lack of studies describing goat production systems in Santa Elena has been the main reason for this study; which consisted in the collection of primary information by applying surveys to livestock farmers of all the communes of the province; which were analyzed by descriptive statistics, where a total of 497 cattle ranchers were surveyed, which had a total of 7293 animals, $75 \%$ female animals, which shows a good relation of the composition of the herd, which, in addition, is conformed with $92 \%$ of native goats. The analyzed variables are related to the current production of goats and were grouped in several components that are, social component, herd composition, existing technology, characteristics of the feeding, animal health and commercialization.
\end{abstract}

Palabras Claves: Livestock, Cattle, Production, Goats. 


\section{Introducción}

Según Bedotti (2008) la domesticación de las cabras lleva ya casi 8000 años A.C en las montañas de Zagros en Iran, y fue el segundo animal en ser domesticado después del perro; se dice que es el primer animal destinado para el consumo humano. En aquellos tiempos la importancia no solo era para alimentación, supervivencia, y de interés religioso, sino también como objeto mitológico.

De las cabras se pueden aprovechar la carne, la leche, la piel y el estiércol; a nivel mundial estos animales contribuyen a la alimentación del hombre en pequeñas cantidades en relación con otros rumiantes como ovinos y bovinos. Aunque, según el Ministerio de Agricultura, Pesca, Alimentación y Medio Ambiente de España, menciona que la producción de cabra a nivel mundial está en constante crecimiento (Ministerio de Agricultura, Pesca, Alimentacion y Medio Ambiente, 2017).

Para Wadsworth, (1997) los animales domesticados que contribuyen a la alimentación de los seres humanos deben ser confinados en fincas, granjas o haciendas para lograr buenos rendimientos, con la finalidad de ser manejados como un sistema, donde se pueda controlar los ingresos (input) y resultados (output), pudiendo así determinar pérdidas o ganancias de un emprendimiento manejado como sistema.

Para interpretar los resultados que tiene un determinado sistema, es necesaria una descripción de sus características, que en muchos casos significa estudiar cada uno de los factores que interviene de una u otra forma en el sistema de producción, ya que los rendimientos no solo están ligados a las características genéticas, sino también a la alimentación, instalaciones, labores de manejo e interacción de estos con el medio ambiente (Cruz, Rodriguez, Benavides, \& Clavijo, 2013).

En el país, el consumo de cabra en algunas localidades forma parte de la cultura gastronómica de la zona, a tal punto que se elaboran platos típicos a partir de ésta, que son ofrecidos principalmente en lugares donde se produce. La producción de caprinos en el país no genera divisas como la producción de pollo, cerdo o bovinos, y debido a su poca influencia sobre el PIB nacional, no se han realizado suficientes estudios al respecto, es por ello que al menos en la península de Santa Elena son pocos los estudios que en esta especie se evidencian (Chevez, 2013).

Con la finalidad de conocer los sistemas de producción caprina que se manejan en la provincia de Santa Elena, se plantea el análisis de los sistemas con una perspectiva más acertada de los principales factores que influyen sobre la producción; generando información básica útil para el planteamiento posterior de soluciones y mejoras de los procesos a través de la elaboración de proyectos de inversión, investigación y/o de desarrollo social necesarios para mejorar las condiciones de vida de los productores caprinos.

\section{Materiales y Métodos}

\subsection{Ubicación y descripción de la zona de estudio.}

Santa Elena, Salinas, y La Libertad son los tres cantones que pertenecen a la provincia de Santa Elena, entre ellos suman $3763,8 \mathrm{~km}^{2}$ de superficie, en donde Santa Elena es el cantón que posee el $97 \%$ del total de la superficie, seguido de Salinas y La Libertad con la menor superficie. El clima de esta región está influenciado por la corriente cálida Del Niño, que llega desde panamá para los meses de diciembre hasta abril y la corriente fría de Humboldt que lo hace desde mayo a noviembre (Prefectura Santa Elena, 2009).

Las temperaturas anuales van de 23 a 25 grados centígrados, con una mínima de 15 para los meses de julio y agosto; registrando la máxima temperatura entre febrero y marzo (39,5 centígrados). Las tierras cultivables y explotables con fines pecuarios se encuentran en la zona rural donde el $80 \%$ del territorio provincial está configurado por la presencia de 68 comunas de las cuales la totalidad de ellas fueron intervenidas para este estudio (Tuaza castro \& Sáenz Ozaetta, 2013).

\subsection{Metodología}

Con la finalidad de intervenir a casi la totalidad de tenedores de ganado caprino de la provincia de Santa Elena, se utilizaron mapas donde se sectorizó primero en parroquias y luego en comunas, siendo un total de 9 parroquias y 68 comunas las que formaron parte del proyecto (Prefectura Santa Elena, 2009).

Para caracterizar los sistemas de producción caprinos de Santa Elena, se utilizó la encuesta como herramienta para el levantamiento de información primaria, la misma que fue estructurada con modelos de la universidad de Córdoba de España; modelo de encuesta que clasifica la información recolectada por componentes para su tabulación (Castalado, 2003).

Para conocer la ubicación de los corrales se recurrió a dirigentes comunales que fueron encuestados; organizando la información de campo con la ayuda de hojas electrónicas de Excel, y para su análisis se utilizó la estadística descriptiva (Villacres Matias \& Bacilio Baquerizo, 2015). 


\subsection{Variables de Estudio}

La información recolectada fue agrupada en 5 componentes que son, componente social, composición del hato, alimentación, sanidad animal, tecnología existente, y comercialización.

\subsubsection{Componente Social}

Dentro de este componentes se consideró servicios básicos, años en la comunidad, y características de la vivienda; además se consideró características de la finca, donde se pregunta sobre la tenencia de la tierra, uso del suelo agrícola y especies agrícolas cultivadas.

\subsubsection{Composición del Hato}

Se describe el motivo de la explotación, razas de preferencia, número de animales por categoría, continuidad en la actividad, preferencia de los ganaderos por nuevos animales, técnicas de reproducción, manejo reproductivo del hato, características de selección y causas del descarte.

\subsubsection{Componente Alimentación}

En este componente se cuestiona sobre el pastoreo y tipos de pastoreo, frecuencia de pastoreo, lugar de pastoreo y especies fitogenéticos que contribuyen a la alimentación de las cabras.

\subsubsection{Componente Sanidad Animal}

Este componente describe la asistencia técnica, aplicación de medicamentos preventivos, biológicos aplicados, y causas de muerte.

\subsubsection{Componente Tecnologías Existentes}

Se cuestiona en este componente sobre las capacitaciones recibidas, gastos promedios de finca, infraestructura de riego, tenencia de botiquines veterinarios y características de los apriscos.

\subsubsection{Componente comercialización}

En este componente se pregunta sobre los canales de comercialización, animales comercializados, y precios percibidos por cada animal.

\section{Resultados y Discusión}

\subsection{Componente social}

Los cuadros 1, 2 y 3 describen las características de la parte social y el entorno en el que se desarrolla el ganadero y su familia, valores presentados tanto en número como en porcentajes.
Cuadro 1. Situación familiar de los capricultores de la provincia de Santa Elena

\begin{tabular}{lrr}
\hline Situación familiar & Total & Porcentaje \\
\hline \hline Estado civil & & \\
Casado & 345 & 69 \\
Separado & 7 & 1 \\
Divorciado & 0 & 0 \\
Soltero & 78 & 16 \\
Unión Libre & 35 & 7 \\
Viudo & 32 & 6 \\
Total & 497 & 100 \\
\hline Composición familiar & & \\
Poseen hijos & 299 & 86 \\
Promedio hijos & 4 & \\
Promedio habs/hogar & 3 & \\
\hline Nivel de educación & & 90 \\
Primaria & 399 & 1 \\
Secundaria & 47 & 9 \\
Universidad & 5 & 100 \\
Analfabeta & 46 & \\
Total & 497 &
\end{tabular}

En el cuadro 1, de los 497 ganaderos encuestados, 345 , es decir el $69 \%$ manifestó estar casado, el 16\% solteros, la unión libre y los viudos poseen valores semejantes, 7 y 6 respectivamente, existiendo una desproporción con lo establecido por el Instituto Nacional de Estadísticas y Censo 2017, que manifiesta que los casados son el 40 y los solteros el $34 \%$ de los que poseen un estado conyugal. El $86 \%$ de los encuestados poseen hijos, y el promedio de hijos por hogar es de 4, mientras que el promedio de habitantes por hogar es igual a 3 .

Con respecto a la preparación, la mayor cantidad de capricultores (80\%) solo posee un nivel primario, solo el $1 \%$ posee estudios universitarios y existe en Santa Elena un $9 \%$ de ganaderos analfabetos, lo que supera al 5,6\%, promedio general de la provincia de Santa Elena (Instituto Nacional de Estadisticas y Censo , 2017).

El cuadro 2, indica que el $94 \%$ de los tenedores de caprinos pose electricidad y por esta cancela un valor promedio de \$ 11; el agua de red cubre al $89 \%$ de la población encuestada, valor que está mejor que el promedio provincial que es 76,1 como lo manifiesta Ecuador en Cifras (2017); de esta solo el 35\% manifestó que es permanente. El costo promedio del $\mathrm{m}^{3}$ en estas localidades es de $\$ 0,4$. Tanquero y agua de pozo se convierten en una opción para el $70 \%$ de los que no poseen agua de red. 
Cuadro 2. Servicios básicos de los capricultores

\begin{tabular}{lrr}
\hline \multicolumn{1}{c}{ Servicios básicos } & Total & Porcentaje \\
\hline \hline Electricidad & & \\
$\mathrm{Si}$ & 467 & 94 \\
$\mathrm{No}$ & 30 & 6 \\
Total & 497 & 100 \\
Gasto promedio (\$) & 11 & \\
\hline Agua & & \\
Posee agua de red & 442 & 89 \\
No posee agua de red & 55 & 11 \\
Permanente & 155 & 35 \\
Transitoria & 88 & 20 \\
Limitada & 195 & 44 \\
Por temporada & 4 & 1 \\
Promedio mensual $\left(\mathrm{m}^{3}\right)$ & 13 & \\
Costo promedio/m & \\
\hline Otras fuentes de agua & 0,4 & \\
Canal & & \\
Río & 4 & 3 \\
Pozo de agua & 19 & 12 \\
Reservorio & 60 & 38 \\
Tanquero & 18 & 11 \\
Otros & 51 & 32 \\
Total & 7 & 4 \\
\hline
\end{tabular}

Cuadro 3. Características de la vivienda capricultores de Santa Elena

\begin{tabular}{lrr}
\hline Tipos de vivienda & Total & Porcentaje \\
\hline \hline Caña - madera & 56 & 11 \\
Madera - cemento & 151 & 30 \\
Hormigón armado & 290 & 58 \\
Total & 497 & 100 \\
\hline Edad del bien & & \\
1 a 10 & 121 & 24 \\
10 a 20 & 140 & 28 \\
20 a 30 & 102 & 21 \\
30 a 40 & 71 & 14 \\
40 a 50 & 46 & 9 \\
50 a 60 & 17 & 3 \\
Total & 497 & 100 \\
\hline
\end{tabular}

El Cuadro 3, menciona que 290 ganaderos es decir el $58 \%$ poseen viviendas de hormigon armado, el $30 \%$ de estructuras entre madera y cemento, y el restante $(11 \%)$ posee casa donde la caña es el principal elemento de construcción. El $49 \%$ de las viviendas posen entre 10 y 30 años, las construcciones nuevas (1 a 10 años) representan el $24 \%$ de las casas.

\subsection{Componente Superficie y Tenencia de Tierra}

La información que responde a la tenencia de la tierra se detalla en el cuadro 5 y 6 , el cual brinda la superficie, tenencia y usos que les dan a las tierras cultivables, cantidades expresadas en número y en términos porcentuales.

Cuadro 4. Superficie y Tenencia de tierra en capricultores

\begin{tabular}{lrr} 
& & \\
\cline { 2 - 3 } Total, superficie & Total & Porcentaje \\
\hline 0,1 a 0,4 & 247 & 50 \\
0,5 a 2 & 183 & 37 \\
2,1 a 5 & 37 & 7 \\
5,1 a 10 & 18 & 4 \\
10,1 a 20 & 7 & 1 \\
20,1 a 50 & 4 & 1 \\
50,1 a 100 & 1 & 0 \\
Más de 100 & 0 & 0 \\
Total & 497 & 100 \\
\hline Tenencia de tierra & & \\
Ganaderos sin & 122 & 28 \\
terrenos & 105 & 22 \\
Propias & 6 & 1 \\
Arrendadas & 264 & 100 \\
Comunal & 497 & \\
Total & 5 & \\
\hline Costo promedio (\$) & 5 & \\
\hline
\end{tabular}

Cuadro 5. Uso del suelo agrícola

\begin{tabular}{lrr}
\hline Uso del suelo agrícola & Total & Porcentaje \\
\hline \hline Pasto naturales & 14 & 4 \\
Cultivos de cereales & 69 & 20 \\
Rastrojos & 14 & 4 \\
Barbechos & 138 & 40 \\
Bosque & 17 & 5 \\
Frutales & 45 & 13 \\
Maderables & 14 & 4 \\
Cultivos hortícolas & 21 & 6 \\
Otros & 14 & 4 \\
Total & 345 & 100 \\
\hline
\end{tabular}

La cantidad de terrenos que poseen los ganaderos en sus explotaciones, en un $50 \%$ refleja superficies inferiores a 0,4 hectáreas, el $48 \%$ de los ganaderos poseen entre 0,5 y 10 has, el $2 \%$ restante se ubica en 
terrenos superiores a las 10 hectáreas. El 28\% no poseen terrenos, el $22 \%$ si posee, y el $49 \%$ cancelan anualmente un promedio de 5 dólares por hectárea y por año a la comuna respectiva; valor que está muy alejado de lo que estiman Tuaza castro \& Sáenz Ozaetta, (2013), que argumentan que el $80 \%$ de las teirras de la provincvia de Santa Elena estan configuradas a manera de comunas; solo el $1 \%$ arrienda tierras.

Un gran número de ganaderos (40\%) mantiene sus tierras en barbecho, un $47 \%$ cultiva sus tierras con cereales, frutales, hortalizas y otros cultivos; solo el $4 \%$ posee pastos naturales, tal como lo describe el Gobierno Autonomo Descentralizado Municipal de Santa Elena, (2014), quien describe que en la provincia la superficie de pastizales no supera el $6,2 \%$ de las superficie.

\subsection{Componente composición del hato}

En el cuadro 6, se presentan motivos por los que el ganadero mantiene la explotación; tipos de razas y/o cruces genéticos, además se explica los criterios de selección de animales que tiene la explotación.

Cuadro 6. Preferencia racial de capricultores

\begin{tabular}{lrr}
\hline \multirow{2}{*}{ Razón de la explotación } & \multicolumn{2}{c}{ Total Santa Elena } \\
\cline { 2 - 3 } & Número & Porcentaje \\
\hline Herencia & 239 & 48 \\
Donación & 40 & 8 \\
Negocio & 209 & 42 \\
Mixto & 10 & 2 \\
Total & 497 & 100 \\
\hline Razas/Cruces genéticos & & \\
Criolla & 459 & 92 \\
Anglo Nubia & 30 & 6 \\
Mestizo & 11 & 2 \\
Boer & 1 & 0,2 \\
Total & 501 & 100 \\
\hline Criterios de selección & & \\
Rusticidad & 264 & 28 \\
Resistencia & 347 & 37 \\
Facilidad de manejo & 338 & 36 \\
Total & 949 & 100 \\
\hline & &
\end{tabular}

El principal motivo por el cual los encuestados poseen cabras es la herencia con un $48 \%$, mientras que un valor cercano $(42 \%)$, se dedica a esta actividad por negocio; 38 productores es decir el $8 \%$ posee animales por donaciones. La raza criolla frecuenta el $92 \%$ de los predios donde se crían cabras y el 6\% la raza Anglo
Nubia, tal como lo argumentan Pesántes \& Hernández(2014), quienes manifiestan que en la region costa las razas mas frecuentes son Criolla y Aglo Nubian. La preferencia de las razas se da en un $37 \%$ por la resistencia, el $36 \%$ indica que es por la facilidad en el manejo, mientras que el $28 \%$ menciona que es por la rusticidad, información que está acorde a los expresado por Contreras \& Meneses (2005), que destacan que las cabras criollas poseen excelente capacidad de rusticidad y facilidad de adaptación a diferentes ambientes.

\begin{tabular}{lrr}
\multicolumn{2}{c}{ Cuadro 7. Composición del Hato Caprino } \\
\hline \multirow{2}{*}{ Composición del hato } & \multicolumn{2}{c}{ Total, Santa Elena } \\
\cline { 2 - 3 } & Número & Porcentajes \\
\hline Hembras & 4046 & 55 \\
Reposición o jóvenes & 1393 & 19 \\
Machos & 692 & 9 \\
Reposición o jóvenes & 869 & 12 \\
Crías & 293 & 4 \\
Total de cabezas & 7293 & 100 \\
\hline Precio (\$/animal) & 55 \\
\hline
\end{tabular}

Al referirnos a la composición del hato (Cuadro 7), en toda Santa Elena existen 7293 animales, valor que está por encima de lo estimado por la Encuesta de Superficie y Produccion Agropecuaria Continua ESPAC, (2015), quien expone que son 2221 los ejemplares de esta especie que posee Santa Elena. De los caprinos de Santa Elena, el 55\% corresponde a hembras en etapa de reproducción, y 19\% a hembras jóvenes, sumando ambas dan un $74 \%$, lo que está acorde a lo evaluado por Cobos \& Aguirre, (2012) quienes argumentan que las hembras deben superar el $71 \%$ del total de los animales del hato para asegurar la reproducción de los mismos. Los machos frecuentan el $21 \%$ de los corrales y las crías representan el $4 \%$ de la población total de caprinos.

Cuadro 8. Preferencia de los productores por nuevos animales

\begin{tabular}{lcc}
\hline \multicolumn{1}{c}{ Razas } & Total & Porcentaje \\
\hline \hline Criolla & 348 & 70 \\
Anglo Nubia & 84 & 17 \\
Mestizo & 20 & 4 \\
Saanen & 10 & 2 \\
Alpina & 10 & 2 \\
Andaluzar & 10 & 2 \\
Boer & 15 & 3 \\
Total & 497 & 100 \\
\hline
\end{tabular}

Se consultó a los ganaderos sobre la adquisición de nuevos animales, y el $70 \%$ de ellos manifestó que desearían más animales de tipo criollos, un $17 \%$ manifestó preferencias por la raza Anglo Nubia; los 
mestizos y los Boer son preferidos por el 3\% de los ganaderos.

Cuadro 9. Manejo reproductivo ganado caprino Santa Elena

\begin{tabular}{lccc}
\hline \multicolumn{1}{c}{ Manejo reproductivo } & $\#$ & Mín & Máx \\
\hline \hline Edad de primera cubrición (meses) & 8 & 5 & 12 \\
Hembras cubiertas por macho/año & 6 & 1 & 56 \\
Intervalo entre parto (meses) & 6 & 5 & 12 \\
Promedio parto al año/predio & 4 & 0 & 32 \\
Promedio partos dobles al año & 2 & 0 & 20 \\
Edad de destete (meses) & 5 & 1,0 & 8 \\
Porcentaje de partos al año & 49 & & \\
\hline Partos totales año & 1986 &
\end{tabular}

Los valores de los parámetros reproductivos de los caprinos se presentan en el cuadro 9, donde se destaca que la edad a la primera cubrición se da en promedio a los 8 meses, coincidiendo con el criterio de Espadas de Castro, (2012) que indica que la cubrición se debe realizar entre los 7 a 10 meses dependiendo del peso del animal. En promedio cada macho cubre a 6 hembras, existiendo casos en los que son subexplotados (1) y sobreexplotados (56).

El intervalo entre parto promedio es de 6 meses, valor que es inferior a lo registrado por Garcia, (1997), quien expone que en razas españolas las cabras necesitan alrededor de 7 meses para generar una cria; pero en las condiciones de Santa Elena, existen casos de fincas que demoran un año en producir un cabrito. Además, hay fincas que no logran ni un parto al año, a pesar de que el promedio sea de 4 partos por año. La presencia de partos dobles es notoria en los predios, evidenciándose como promedio anual 2 animales por parto, existiendo también fincas que obtienen hasta 8 partos dobles por año.

La edad al destete promedio de las fincas es a los 5 meses; hay fincas que destinan 8 meses para realizar esta actividad, siendo un valor no adecuado, debido a que según Casaretto, (2010) los cabritos pueden destetarse a partir de los 4 meses. De forma general Santa Elena posee 1986 partos por año y esto representa el $49 \%$ de las hembras adultas en existencia.

\subsection{Componente alimentación del ganado caprino.}

La alimentación de los caprinos en Santa Elena es un factor condicionante para el desarrollo y crecimiento de los rumiantes, es por ello que en este componente se describe el tipo de alimentación, el pastoreo, la frecuencia de pastoreo y los recursos fitogenéticos que sustentan la alimentación de esta especie animal.
Cuadro 10. Tipo de alimentación que se provee a caprinos

\begin{tabular}{lcc}
\hline \multirow{2}{*}{ Tipo de alimentación } & \multicolumn{2}{c}{ Total } \\
\cline { 2 - 3 } & Número & Porcentaje \\
\hline Balanceado & 1 & 0 \\
Residuos de cosecha & 393 & 46 \\
Recursos fitogenéticos autóctonos & 460 & 54 \\
Pastos cultivados & 5 & 1 \\
Total & 859 & 100 \\
\hline
\end{tabular}

El cuadro 10 presenta los tipos de alimentación que se utilizan en la producción de caprinos, donde solo un ganadero utiliza balanceado $(0,11 \%)$, el $54 \%$ utiliza recursos fitogenéticos y el $46 \%$ residuos de cosecha, y solo el $1 \%$ de ganaderos cultivan pastos para sus animales; estos datos están relacionados a que las cabras son animales que poseen buena velocidad digestiva, son capaces de degradar alimentos de mala calidad, y excelentes en aprovechar subproductos de la agricultura, (Jimeno, Rebollar, \& Castro, 2003).

Cuadro 11. Características del pastoreo en caprinos

\begin{tabular}{lcc}
\hline \multirow{2}{*}{ Lugar de pastoreo } & \multicolumn{2}{c}{ Total } \\
\cline { 2 - 3 } & Número & Porcentaje \\
\hline Pastos cultivados & 5 & 0,4 \\
Barbechos & 434 & 33 \\
Pastos naturales & 440 & 33 \\
Rastrojos & 396 & 30 \\
Montañas & 58 & 4 \\
Otros & 0 & 0 \\
Total & 1333 & 100 \\
\hline Tipos de pastoreos & & \\
Libre pastoreo & 328 & 66 \\
Guiado & 169 & 34 \\
Otros & 0 & 0 \\
Total & 497 & 100 \\
\hline Meridiano & Horas \\
Mañana & 3 & \\
Tarde & 4 & \\
Mañana y tarde & 7 & \\
Promedio horas pastoreo & 5 & \\
\hline
\end{tabular}

El $66 \%$ de las cabras pastorean en barbechos o en pastos naturales, un 30\% lo hace en rastrojos, y un $4 \%$ en pastos cultivados (Cuadro 11). Predomina en los ganaderos el libre pastoreo $(66 \%)$ y el $34 \%$ restante guía a sus animales al momento de buscar alimentos. Las horas de pastoreo son 5 como promedio al día, aunque pueden llevarse más horas cuando el pastoreo es en la mañana y tarde ( 7 horas), siendo 3 en la mañana y 4 en la tarde, valor que para Gioffredo \& 
Petryna, (2010), es inferior ya que describen que en sistemas extensivos de explotacion son 8 horas las que el animal necesita para suplir sus necesidades.

Cuadro 12. Especies que contribuyen a la alimentación de caprinos de Santa Elena

\begin{tabular}{lrr}
\hline Residuos de cosecha & Número & Porcentaje \\
\hline \hline Hortalizas & 148 & 25,4 \\
Externos & 39 & 6,7 \\
Cereales & 168 & 28,8 \\
Otros (Hojas de choclos) & 228 & 39,1 \\
Total & 583 & 100 \\
Pastos cultivados & & \\
\hline Si cultiva & 5 & 1 \\
No cultiva & 492 & 99 \\
Total & 497 & 100 \\
Especies fitogéneticas & & \\
\hline Muyuyo & 451 & 12 \\
Cascol & 429 & 11 \\
Niguito & 425 & 11 \\
Algarrobo & 458 & 12 \\
Verdolaga & 368 & 10 \\
Ebano & 310 & 8 \\
Seca & 438 & 12 \\
Pasto natural & 430 & 11 \\
Bejuco de camote & 296 & 8 \\
Otros (Cardón) & 172 & 5 \\
\hline Total & 3777 & 100
\end{tabular}

Dentro de los residuos de cosechas más utilizados en la provincia de Santa Elena para la alimentación de caprinos están, las hojas de choclos con un 39\%, y esto se sustenta con los datos de la Encuesta de Superficie y Produccion Agropecuaria Continua ESPAC, (2015), que indica que son 3637 las hectáreas cultivadas de maíz; valor muy cercano poseen los residuos de cereales $(28,8 \%)$, los residuos de hortalizas con un $25 \%$, y existe un $6,7 \%$ de materias que se obtiene de forma externa (Cuadro 12).

Solo el $1 \%$ de los ganaderos cultivan pastos, por lo que las cabras recurren a las especies fitogenéticas que crecen de forma natural, y que se han identificado que son 10 las que más representativas en la ganadería caprina de esta región, tal como lo Expone Stemmer \& Zárate, (2014) al momento de nombrar al algarrobo y otras especies silvestre como contribuyentes al mantenimiento de la explotación caprina.

\subsection{Componente Sanidad animal}

Dentro de los aspectos sanitarios, los cuadros 13, 14 y 15 , presentan información sobre las principales causas de descarte de los animales; productos y biológicos utilizados en la prevención de patología, así como también las principales enfermedades que causan mortalidad en los caprinos.

Cuadro 13. Causas de descarte en reproductores caprinos

\begin{tabular}{lrr}
\hline \multirow{2}{*}{ Causas de descarte } & \multicolumn{2}{c}{ Total } \\
\cline { 2 - 3 } & Número & porcentaje \\
\hline Producción de leche & 27 & 7 \\
Reproductivos & 101 & 25 \\
Sanitarios & 14 & 3 \\
Locomotores & 2 & 0,5 \\
Otros (necesidad) & 262 & 65 \\
Total & 406 & 100 \\
\hline Hembras & 150 & 47 \\
Machos & 170 & 53 \\
Total & 320 & 100 \\
\hline Edad promedio Hembra (años) & 2 & \\
Edad promedio macho (años) & 2 \\
Precio promedio del animal (\$) & 56 \\
\hline
\end{tabular}

Cuadro 14. Aspectos sanitarios de los caprinos de Santa Elena

\begin{tabular}{lrr}
\hline Asociación & \multicolumn{2}{c}{ Total } \\
Púmero & Porcentaje \\
\hline \hline Pertenece & 489 & 1 \\
No pertenece & 496 & 99 \\
Total & 20 & 100 \\
Asesoría por veterinario & 1 & 0 \\
\hline Frecuencia de visitas al & & 1 \\
año & 140 & \\
Productos & 190 & \\
\hline Vitaminas & 103 & \\
Antiparasitarios & & 66 \\
Vacunas & 40 & 34 \\
Biológicos aplicados & 21 & 0 \\
\hline Triple o polivalente & 0 & \\
Aftosa & 0 & \\
Bronquitis & 61 & \\
Brucelosis & & \\
\hline Total & & \\
\hline
\end{tabular}


Cuadro 15. Causas de mortalidad o perdidas de caprinos de Santa Elena

\begin{tabular}{lrr}
\hline \multicolumn{1}{c}{$\begin{array}{c}\text { Causas de } \\
\text { Mortalidad }\end{array}$} & \multicolumn{2}{c}{ Total } \\
\cline { 2 - 3 } & Número & Porcentaje \\
\hline Procesos diarreicos & 124 & 16 \\
Procesos respiratorios & 60 & 8 \\
Enterotoxemias & 2 & 0 \\
Timpanismo & 15 & 2 \\
Defectos congénitos & 20 & 3 \\
Abortos & 202 & 26 \\
Desnutrición & 38 & 5 \\
Otros & 311 & 40 \\
Total & 772 & 100 \\
\hline
\end{tabular}

En las comunidades intervenidas fueron algunas las causas de descarte; una de las principales son las necesidades económicas (65\%), en segundo lugar están los problemas reproductivos que puedan tener los animales con un $25 \%$, un $7 \%$ descarta a las cabras por baja producción de leche y el $3 \%$ de los animales se los descarta por problemas sanitarios, los machos se descartan en mayor número que las hembras, y la edad de descarte promedio para ambos sexos es de 2 años (Cuadro 13).

El 99\% de los productores de cabra no pertenecen a ninguna asociación, y solo reciben una vez al año la visita de algún veterinario; los antiparasitarios son los insumos veterinarios más utilizados, y respecto a los biológicos que mayormente se aplican, son para contrarrestar la triple bacteriana y la fiebre aftosa, con el $66 \%$ y $34 \%$ respectivamente. Las causas de mortalidad o perdidas se producen en un $26 \%$ por abortos, un $16 \%$ por problemas diarreicos, que de acuerdo a Ministerio de Economia Familiar Comunitaria, Cooperativa y Asociativa, (2015), son muy comunes en los caprinos que no se posee un control en la alimentacion. En un $8 \%$ se descartan animales por problemas respiratorios; pudiéndose evidenciar mayores pérdidas (40\%) por factores externos, que los ganaderos manifestaron son ataques de animales salvajes y robos de ganado.

\subsection{Componente tecnología existente}

Como tecnología existente en la producción de caprinos, se destacan las capacitaciones, los gastos destinados a la producción, y las características de los corrales, (Cuadros 16, 17, 18 y 19).
Cuadro 16. Capacitaciones impartidas a capricultores

\begin{tabular}{lrr}
\hline \multirow{2}{*}{$\begin{array}{l}\text { Asistencia a } \\
\text { Capacitaciones }\end{array}$} & \multicolumn{2}{c}{ Total } \\
\cline { 2 - 3 } & Número & Porcentaje \\
\hline $\mathrm{Si}$ & 42 & 9 \\
$\mathrm{No}$ & 446 & 91 \\
Total & 488 & 100 \\
\hline Quien los capacita & & \\
MAGAP & 32 & 82 \\
UPSE & 2 & 5 \\
Plan Internacional & 1 & 3 \\
CPR & 4 & 10 \\
Total & 39 & 100 \\
\hline
\end{tabular}

Cuadro 17. Actividades económicas de los capricultores

\begin{tabular}{lrr}
\hline \multicolumn{1}{c}{ Actividades } & Número & Porcentaje \\
\hline \hline Agricultor & 131 & 27 \\
Comerciante & 65 & 13 \\
Albañil & 14 & 3 \\
Carpintero & 16 & 3 \\
Pesca & 10 & 2 \\
Docente & 2 & 0 \\
Diversas actividades & 251 & 51 \\
Total & 489 & 100 \\
\hline
\end{tabular}

Cuadro 18. Gastos operaciones de productores

\begin{tabular}{lrr}
\hline \multicolumn{1}{c}{ Suministros } & $\begin{array}{r}\text { Gasto promedio } \\
(\mathbf{\$} / \mathbf{m e s})\end{array}$ & Porcentaje \\
\hline \hline Gasolina & 10 & 37 \\
Electricidad & 9 & 31 \\
Agua & 7 & 25 \\
Telefonía fija & 1 & 5 \\
Insumos & 1 & 2 \\
Sanitarios & & 0 \\
Otros & 0 & 100 \\
Total & 28 & \\
\hline
\end{tabular}

El 91\% de los encuestados no recibe ningún tipo de capacitación, y el $9 \%$ de los que se capacitan lo hacen a través del Ministerio de Agricultura, Ganadería, Acuacultura y Pesca MAGAP (82\%); mientras que los ganaderos de la provincia son capacitados por el Centro de Promoción Rural CPR y la Universidad Estatal Península de Santa Elena UPSE en un $10 \%$ y $5 \%$ respectivamente (Cuadro 16). 
Cuadro 19. Características de los corrales caprinos

\begin{tabular}{lrr}
\hline Tipo de construcción & Número & Porcentaje \\
\hline \hline Fabricado madera rústica & 289 & 78 \\
Fabricado caña guadua & 44 & 12 \\
Fabricado tipo mixta & 15 & 4 \\
Fabricados de maderas trabadas & 20 & 6 \\
Total & 368 & 100 \\
\hline Estado & & \\
Bueno & 121 & 33 \\
Regular & 225 & 61 \\
Malo & 22 & 6 \\
Total & 368 & 100 \\
\hline Higiene de pisos & & \\
Deficiente & 19 & 5 \\
Intermedia & 314 & 85 \\
Buena & 35 & 10 \\
Total & 368 & 100 \\
\hline
\end{tabular}

El $27 \%$ de los productores de cabras tienen a la agricultura como otra fuente de ingresos económicos, el $13 \%$ se dedica a actividades de comercio, $8 \%$ se distribuye entre albañilería, carpintería y pesca, pero un $51 \%$ manifestó que son varias las actividades que realizan para tener un sustento económico diario (Cuadro 17). Los insumos más utilizados son la gasolina, electricidad y agua, con un $37 \%, 31 \%$ y 25 $\%$ de los gastos totales respectivamente (Cuadro 18).

La mayor cantidad de los apriscos están construidos de madera en forma rústica (78\%), el $61 \%$ de ellos están en condiciones regulares $(61 \%)$ y la higiene de los pisos es intermedia (Cuadro 19). En el caso de los biológicos más utilizados para inmunizar los caprinos son para la Aftosa y la Polivalente, tal como lo recomienda La Agencia Ecuatorian del Aseguramiento de la Calidad y El Agro, (2016).

\subsection{Componente Comercialización.}

La comercialización de especies pecuarias poseen particularidades típicas de cada zona, es por ello que en el cuadro 20 y 21 se describen los canales de comercialización, y número de animales comercializados por año y por finca.

El cuadro 20 y 21 demuestra que el $63 \%$ de los ganaderos utilizan intermediarios como medio de comercialización; otro sistema que tiene relevancia es la venta a particulares que compran animales para consumo propio, los animales más comercializados son los machos y el precio promedió percibido por animal es de 56 dólares, es decir ellos perciben $\$ 1,6$ por libra, siendo un precio bajo según periódico La Hora Nacional, (2015), que indica que la carne de caprino se comercializa a $\$ 2,75 /$ libra. Cabe recalcar que las ventas son escasas en las fincas pudiéndose evidenciar un animal como promedio de venta anual por finca.

Cuadro 20. Canales de comercialización de caprinos

\begin{tabular}{lrr}
\hline \multicolumn{1}{c}{ Canales } & Número & Porcentaje \\
\hline \hline Intermediario & 235 & 63 \\
Carnicero & 11 & 3 \\
Particular & 108 & 29 \\
Carnicería propia & 18 & 5 \\
Total & 372 & 100 \\
\hline
\end{tabular}

Cuadro 21. Animales comercializados por fincas al año

\begin{tabular}{lcc}
\hline \multicolumn{1}{c}{ Animales } & Número & Porcentajes \\
\hline \hline Hembras & 175 & 47 \\
Machos & 197 & 53 \\
Total & 372 & 100 \\
\hline Precio animal $(\$)$ & & 56 \\
\hline
\end{tabular}

\section{Conclusiones y Recomendaciones}

\section{Conclusiones:}

Los animales caprinos que más frecuentemente se encuentran en las fincas ganaderas son los criollos, que son preferidos por su facilidad de manejo como por su rusticidad. La actividad caprina, en términos generales constituye una actividad de subsistencia que en la totalidad de los casos es una alternativa a otras actividades económicas.

A pesar de que, las cabras poseen buena adaptación al ambiente de Santa Elena, repercutiendo positivamente sobre la reproduccion, sus parámetros reproductivos no están acordes a los estándares internacionales, atribuyéndose esto a varios factores entre los cuales citamos los siguientes: la primera cubrición de las cabras en Santa Elena se da hasta 4 meses antes de lo recomendado; un caprino macho a nivel mundial puede cubrir 25 hembras, en este estudio el promedio es solo 6; a nivel mundial los caprinos se destetan a los 3 meses, en Santa Elena necesitan 5; y el porcentaje de abortos produce el $25 \%$ de las pérdidas que en óptimas condiciones ese valor no debe superar el 5\%. Todos estos parámetros están ligados al manejo que el ganadero da al caprino, valores que no son los óptimos, pudiendo concluir que en Santa Elena 
la cabra esta sub explotada y su potencial genético no está expresado en su totalidad.

Son diez especies fitogenéticos que mantienen a la ganadería caprina de Santa Elena, que se constituyen en el recurso fitogenéticos silvestre de la zona.

Existen falencias en el proceso de comercialización de las cabras, ya que en su mayoría se da por medio de intermediarios que solo ofrecen 56 dólares por animal adulto, valor que no es representativo para los 24 meses de periodos de crianza.

\section{Recomendaciones:}

Esta información puede ser utilizada como línea base para futuros proyectos que se generen en el ámbito social, de investigación, de inversión.

Considerando que especies fitogenéticos aquí identificadas son apetecidas por las cabras para su alimentación, es necesario que los ganaderos planteen cultivos de estas especies con fines de nutrición animal así como de conservación de las especies.

Extender capacitaciones a los productores de ganado caprino que permitan mejorar los sistemas de producción; plantear además dietas para caprinos utilizando los recursos fitogenéticos y materias primas de la zona.

Implementar pastizales cultivados con la finalidad de cambiar de un sistema extensivo a sistemas tabulados donde se optimicen los nutrientes ingeridos por las cabras.

\section{Referencias}

Agencia Ecuatorian del Aseguramiento de la Calidad y El Agro. (14 de Diciembre de 2016). Agrocalidad. Obtenido de http://www.agrocalidad.gob.ec/iniciacampana-de-vacunacion-contra-la-fiebre-aftosa/

Bedotti, F. (2008). El Rol Social del ganado caprino. 31 congreso Argentino de Produccion Animal (págs. http://www.produccion-

animal.com.ar/produccion_caprina/produccion_capri na/11-rol_social.pdf). San luis: Sitio Argentino de Produccion Animal.

Casaretto, A. (13 de mayo de 2010). El Destete. Argentina: Sitio Argentino de Produccion Animal.

Castalado, A. O. (2003). Caracterizacion de los sistemas de produccion bovina (invernada) en el noroeste de la provincia de la Paampa (argentina). Cordoba: Universidad de Cordoba.
Chevez, J. (2013). Caracterizacion morfologica y faneroptica de las razas caprinas en la provincia de Santa Elena. Quevedo: Universidad .

Cobos, E., \& Aguirre, G. (2012). Caracterización de la administración y la estructura del costo de produccion en cabras en la parroquia Limones del cantón Zapotillo, para diseñar un plan crediticio que desarrolle sosteniblemente esta exoplotación. Loja, Ecuador: Universidad Nacional de Loja.

Contreras, C., \& Meneses, R. (2005). Razas ovinas y caprinas en el instituto de investigaciones Agropecuarias. Chile: INIA.

Cruz, J., Rodriguez, D., Benavides, A., \& Clavijo, J. (2013). Caracterizacion de parámetros productivos y reproductivos del ganado normando en Colombia. Scielo, 15.

Ecuador en Cifras. (26 de abril de 2017). Resultados del censo 2010. Obtenido de http://www.ecuadorencifras.gob.ec/wpcontent/descargas/Manu-lateral/Resultadosprovinciales/santa_elena.pdf

Encuesta de Superficie y Produccion Agropecuaria Continua ESPAC. (2 de Mayo de 2015). Ecuador en Cifras. Obtenido de http://www.ecuadorencifras.gob.ec//documentos/web -inec/Estadisticas_agropecuarias/espac/espac_20142015/2015/Presentacion\%20de\%20resultados\%20ES PAC_2015.pdf

Espadas de Castro, M. (Septiembre de 2012). Razas de Ganado Caprino. Menorca, España: Remugants.

Garcia, A. (1997). Intervalo entre partos en ganado caprino. Mundo Ganadero, 1-3.

Gioffredo, J., \& Petryna, A. (abril de 2010). Caprinos: Generalidades, Nutrición, Reproducción, e Instalaciones. Rio Cuarto, Argentina: Sitio Argentino de Produccion animal.

Gobierno Autonomo Descentralizado Municipal de Santa Elena. (2014). Plan de desarrollo y ordenamiento territorial del canton Santa Elena 2014-2019. Santa Elena: GAD Santa Elena.

Instituto Nacional de Estadisticas y Censo . (26 de Abril de 2017). Ecuador en cifras. Obtenido de http://www.ecuadorencifras.gob.ec/wp- 
content/descargas/Manu-lateral/Resultados-

provinciales/santa_elena.pdf

Jimeno, V., Rebollar, P., \& Castro, T. (2003). Nutricion y alimentacion del caprino de leche en sistemas intensivos de explotacion. XIX Cuerso de Especializacion FEDNA (pág. 24). Madrid: FEDNA.

La Hora Nacional. (18 de agosto de 2015). La hora nacional. Obtenido

de

http://lahora.com.ec/index.php/noticias/show/110185 3905/-

1/Fijan_el_precio_de_la_carne_de_chivo.html\#.WQJ L11U1_IU

Ministerio de Agricultura, Pesca, Alimentacion y Medio Ambiente. (12 de Abril de 2017). Ministerio de Agricultura, Pesca, Alimentacion y Medio Ambiente. Obtenido

de http://www.mapama.gob.es/es/ministerio/servicios/in formacion/plataforma-de-conocimiento-para-elmedio-rural-y-pesquero/observatorio-de-tecnologiasprobadas/sistemas-prodnut-animal/ganado-

caprino.aspx\#para1

Ministerio de Economia Familiar Comunitaria, Cooperativa y Asociativa. (21 de Septiembre de 2015). MEFCCA. Obtenido de http://cdoc.economiafamiliar.gob.ni/2015/09/21/enfe rmedades-del-ganado-caprino/

Pesántes, M., \& Hernández, A. (2014). Produccion Lechera de cabras Criollas y Anglo-Nubian en Loja, Ecuador. Revista Cubana de Ciencias Agricolas, 2-4.

Prefectura Santa Elena. (29 de Octubre de 2009).

Santa Elena Prefectura. Obtenido de http://www.santaelena.gob.ec/index.php/santa-elena
Stemmer, A., \& Zárate, A. (2014). Cabras criollas y pequeños productores: caminos para elevar la productividad, experiencias del proyecto caprinos en Cochabamba-bolivia. Actas Iberoamaericanas de conservacion animal (pág. 183). Bolivia: AICA.

Tuaza castro, L., \& Sáenz Ozaetta, c. (2013). Federacion de comunas de la provincia de Santa Elena: Capacidad de Agencia y Conflictos. Ciencias Pedagogicas e innovacion, 1-5.

Tuaza, L. (2014). Federacion de las comunas de la provincia de Santa Elena: capacidad de de agencia y conflictos. Ciencias Pedagógicas e innovación, 2528.

Villacis, B. (23 de abril de 2017). Instituto Nacional de Estadisticas y censo. Obtenido de http://www.inec.gob.ec/inec/index.php?option=com content $\&$ view $=$ article $\& i d=422 \% 3$ Ase-reduce-eltamano-de-los-hogaresecuatorianos $\&$ catid $=68 \% 3$ Aboletines $\&$ Itemid $=51 \&$ la ng=es

Villacrés Matias, J., \& Bacilio Baquerizo, B. (2015). Estudio socio economico de la ganaderia caprina (Capra hircus) en la zona norte de la parroquia Colonche, cantón Santa Elena. Santa Elena, Santa Elena, Ecuador: Repositorio Universidad Estatal Peninsula de Santa Elena.

Wadsworth. (1997). Anailis de sistemas de produccion Animal. Roma: Organizacion Mundia para la Agricultura y Alimentacion FAO. 